\title{
The Larger Bound on the Domination Number of Fibonacci Cubes and Lucas Cubes
}

\author{
Shengzhang $\operatorname{Ren}^{1,2}$ \\ ${ }^{1}$ School of Mathematics and Computer Science, Shaanxi University of Technology Hanzhong, Shaanxi 723001, China \\ ${ }^{2}$ Department of Mathematics, Lanzhou University Lanzhou, Gansu 730000, China
}

Correspondence should be addressed to Shengzhang Ren; renshengzhang1980@163.com

Received 18 September 2013; Revised 9 January 2014; Accepted 19 January 2014; Published 4 March 2014

Academic Editor: Yuantong Gu

Copyright (c) 2014 Shengzhang Ren. This is an open access article distributed under the Creative Commons Attribution License, which permits unrestricted use, distribution, and reproduction in any medium, provided the original work is properly cited.

Let $\Gamma_{n}$ and $\Lambda_{n}$ be the $n$-dimensional Fibonacci cube and Lucas cube, respectively. Denote by $\Gamma\left[u_{n, k, z}\right]$ the subgraph of $\Gamma_{n}$ induced by the end-vertex $u_{n, k, z}$ that has no up-neighbor. In this paper, the number of end-vertices and domination number $\gamma$ of $\Gamma_{n}$ and $\Lambda_{n}$ are studied. The formula of calculating the number of end-vertices is given and it is proved that $\gamma\left(\Gamma\left[u_{n, k, z}\right]\right) \leq 2^{k-1}+1$. Using these results, the larger bound on the domination number $\gamma$ of $\Gamma_{n}$ and $\Lambda_{n}$ is determined.

\section{Introduction}

The Fibonacci cube and Lucas cube were presented in $[1,2]$, respectively. Because their many properties (see [1-7]) such as domination number, 2-packing number, and observability can be applied to interconnection networks [1].

However, the number of vertices of Fibonacci cube $\Gamma_{n}$ and Lucas cube $\Lambda_{n}$ grows rapidly as $n$ increases. So it is hard to calculate exactly the number of domination number of Fibonacci cubes and Lucas cubes. The lower bound on the domination number of Fibonacci cubes and Lucas cubes is determined in $[3,6]$, respectively. In this paper, we will give a larger bound on the domination number of Fibonacci cubes and Lucas cubes using construction method. We begin with some basic definitions.

Graphs considered in this paper are finite, simple, connected, and undirected. Let $Q_{n}$ be the $n$-dimensional hypercube with $n>0$. A Fibonacci string $b_{1} b_{2} \cdots b_{n}$ of order $n$ is a binary string of length $n$ without two consecutive ones. The Fibonacci cube $\Gamma_{n}$ (see Figure 1) is the subgraph of $Q_{n}$ induced by the Fibonacci strings of length $n$, whose vertices are the Fibonacci strings of length $n$, and two vertices are joined by an edge if their Hamming distance is exactly 1 . A Fibonacci string $b_{1} b_{2} \cdots b_{n}$ is a Lucas string if $b_{1} b_{n}=0$. The Lucas cube $\Lambda_{n}$ is the subgraph of $Q_{n}$ induced by the Lucas strings of length $n$. It is well known that $\left|V\left(\Gamma_{n}\right)\right|=F_{n+2}$ and $\left|V\left(\Lambda_{n}\right)\right|=L_{n}$, where $F_{n}$ and $L_{n}$ are Fibonacci numbers and Lucas numbers, respectively. Recall that the Fibonacci numbers and Lucas numbers form a sequence of positive integers $F_{n}$ and $L_{n}$, respectively, where $F_{1}=1, F_{2}=1$, and $F_{n}=F_{n-1}+F_{n-2}$ and $L_{1}=1, L_{2}=3$, and $L_{n}=L_{n-1}+L_{n-2}$ for $n>2$.

For a connected graph $G$, the distance $d_{G}(u, v)$ between vertices $u$ and $v$ is the usual shortest path distance. For $0 \leq$ $k \leq n, n \geq 1$, let $\Gamma_{n, k}$ be the set of vertices of $\Gamma_{n}$ that contain $k$ ones. Hence $\Gamma_{n, k}$ is the set of vertices of $\Gamma_{n}$ at distance $k$ from $b_{1} b_{2} \cdots b_{n}, b_{i}=0,1 \leq i \leq n . \Lambda_{n, k}$ is defined analogously. If $u v \in \Gamma_{n}$, where $u \in \Gamma_{n, k}$ and $v \in \Gamma_{n, k-1}$ for $k \geq 1$, then we call $v$ a down-neighbor of $u$ and $u$ an up-neighbor of $v$.

If a vertex $u$ has no up-neighbor, we call it an end-vertex and denote by $\sigma\left(\Gamma_{n}\right)$ the number of end-vertices of $\Gamma_{n}$. Let $u_{n, k, z}(0 \leq k \leq n / 2, z \geq 0, n \geq 1)$ be an end-vertex with string length $n$, where $k, z$ are the number of ones and consecutive $0^{2}$, respectively. We denote by $\Gamma\left[u_{n, k, z}\right]$ the subgraph of $\Gamma_{n}$ induced by the end-vertex $u_{n, k, z}$, whose strings of vertices were obtained from string of the vertex $u_{n, k, z}$ by changing $i$ ones into $i$ zeroes $(i=0,1,2, \ldots, k)$ and any two vertices have an edge if their Hamming distance is exactly 1 (see Figure 2).

Let $G$ be a graph. Then $D \subseteq V(G)$ is a dominating set if every vertex from $V(G) \backslash D$ is adjacent to some vertex from $D$. The domination number $\gamma(G)$ is the minimum cardinality of a dominating set of $G$. 


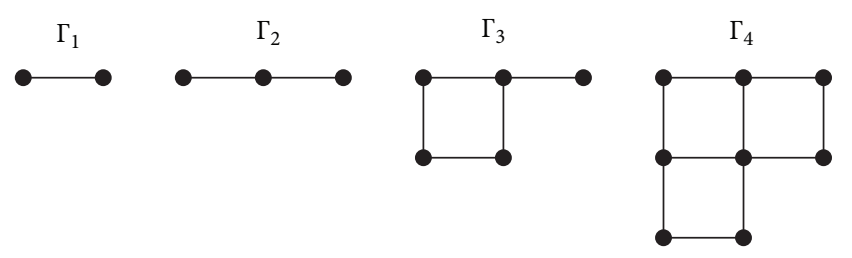

Figure 1: $\Gamma_{1}, \Gamma_{2}, \Gamma_{3}$, and $\Gamma_{4}$.

All graph-theoretical terms and concepts used but unexplained in this paper are standard, and that can be found in many textbooks such as [8].

\section{The Larger Bound on the Domination Number of Fibonacci Cubes}

In this section, we will determine the larger bound on the domination number of Fibonacci cubes. Firstly, we mention the following properties of Fibonacci cubes which will be used later.

Lemma 1 (see [3]). Let $n \geq 3$ and $k \geq 2$. Then any different $u, v \in \Gamma_{n, k}$ have different sets of down-neighbors.

Lemma 2 (see [6]). For any $n \geq 4, \gamma\left(\Gamma_{n}\right) \geq\left\lceil\left(F_{n}-3\right) /(n-2)\right\rceil$.

Lemma 3. Let $u_{n, k, z}$ be an end-vertex with string length $n$, where $k$ and $z$ are the number of ones and consecutive $0^{2}$, respectively. Then $\gamma\left(\Gamma\left[u_{n, k, z}\right]\right) \leq 2^{k-1}+1$ (see Figure 2).

Proof. Let $C_{n}^{m}=(n \cdot(n-1) \cdots(n-m+1)) /(m \cdot(m-1) \cdots 2 \cdot 1)$, and it will be frequently used in latter. Let $u=u_{n, k, z}$, and $v_{h}^{j}$ be a vertex with $d_{\Gamma\left[u_{n, k, z}\right]}\left(u, v_{h}^{j}\right)=j\left(1 \leq h \leq C_{k}^{j}, j=1,2, \ldots, k\right)$, and

$$
\begin{aligned}
\Phi=\left\{u, v_{1}^{1}, v_{2}^{1}, \ldots, v_{\left\lfloor C_{k}^{1} / 2\right\rfloor}^{1}, v_{1}^{2}, v_{2}^{2}, \ldots,\right. \\
\\
\left.v_{\left\lfloor C_{k}^{2} / 2\right\rfloor}^{2}, \ldots, v_{1}^{k-1}, v_{2}^{k-1}, \ldots, v_{\left\lfloor C_{k}^{k-1} / 2\right\rfloor}^{k-1}, v_{1}^{k}\right\},
\end{aligned}
$$

whose subset $\left\{v_{1}^{i}, v_{2}^{i}, \ldots, v_{\left[C_{k}^{i} / 2\right\}}^{i}\right\}(i=2,3, \ldots, k-1)$ contains all vertices $\left\{v_{h}^{i} \mid 1 \leq h \leq C_{k}^{i}\right\}$ such that satisfy the condition $d_{\Gamma\left[u_{n, k, z}\right]}\left(v_{l}^{i-1}, v_{h}^{i}\right) \neq 1$ for $l=1,2, \ldots,\left\lfloor C_{k}^{i-1} / 2\right\rfloor$. We will prove that $\Phi$ is a dominating set. In order to prove that $\Phi$ is a dominating set. It suffices to prove that any $\left\lfloor C_{k}^{i-1} / 2\right\rfloor$ vertices in $\left\{v_{1}^{i-1}, v_{2}^{i-1}, \ldots, v_{C_{k}^{i-1}}^{i-1}\right\}$ can dominate $\left\lfloor C_{k}^{i} / 2\right\rfloor$ vertices in $\left\{v_{1}^{i}, v_{2}^{i}, \ldots, v_{C_{k}^{i}}^{i}\right\}$. If $C_{k}^{i-1} \geq C_{k}^{i}$, the result is obviously correct. We assume that $C_{k}^{i-1}<C_{k}^{i}$. From Lemma 1, we know that any different $v_{h}^{i-1}, v_{l}^{i-1} \in V\left(\Gamma\left[u_{n, k, z}\right]\right)\left(1 \leq h, l \leq C_{k}^{i-1}, h \neq l\right)$ have different sets of down-neighbors and have at most one common down-neighbor vertex. Since each vertex $v_{h}^{i-1}$ has exactly $k-i+1$ ones. If $k-i+2 \geq\left\lfloor C_{k}^{i-1} / 2\right\rfloor$, then the number

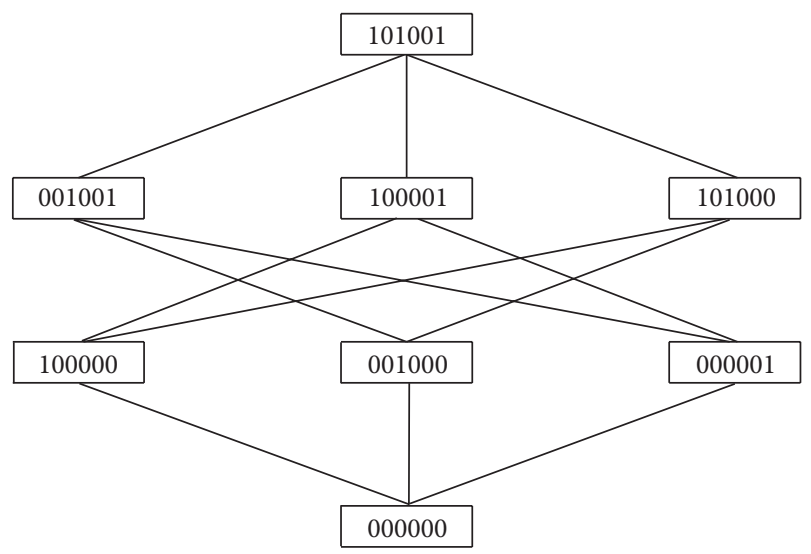

FIGURE 2: $\Gamma\left[u_{n, k, z}\right], u_{n, k, z}=101001$.

of vertices dominated by vertices $\operatorname{set}\left\{v_{1}^{i-1}, v_{2}^{i-1}, \ldots, v_{\left\lfloor C_{k}^{i-1} / 2\right]}^{i-1}\right\}$ is at least as follows:

$$
\begin{aligned}
\sum_{j=1}^{\left\lfloor C_{k}^{i-1} / 2\right\rfloor}(k-i-j+2) & =\left\lfloor\frac{C_{k}^{i-1}\left(2 k-2 i+3-\left\lfloor C_{k}^{i-1} / 2\right\rfloor\right)}{4}\right\rfloor \\
& \geq\left\lfloor\frac{C_{k}^{i-1}(k-i+1)}{4}\right\rfloor \\
& =\left\lfloor\frac{i C_{k}^{i}}{4}\right\rfloor \geq\left\lfloor\frac{C_{k}^{i}}{2}\right\rfloor \quad(\text { Since } i \geq 2)
\end{aligned}
$$

If $k-i+2<\left\lfloor C_{k}^{i-1} / 2\right\rfloor$, there must exist integer $m$ such that $m<\left\lfloor C_{k}^{i-1} / 2\right\rfloor$ and $k-i-m+1=0$, and then the number of vertices dominated by vertices $\operatorname{set}\left\{v_{1}^{i-1}, v_{2}^{i-1}, \ldots, v_{\left\lfloor C_{k}^{i-1} / 2\right\}}^{i-1}\right\}$ is at least as follows:

$$
\sum_{j=1}^{m}(k-i-j+2)=C_{k}^{i}>\left\lfloor\frac{C_{k}^{i}}{2}\right\rfloor .
$$

Therefore the set $\Phi$ is a dominating set, and

$$
|\Phi|=2+\sum_{i=1}^{k-1}\left\lfloor\frac{C_{k}^{i}}{2}\right\rfloor \leq 2^{k-1}+1
$$

This completes our proof.

Theorem 4. Let $\sigma\left(\Gamma_{n}\right)$ be the number of end-vertices in $\Gamma_{n}$. Then the followings hold. 
(i) If $n(n=2 p+1)$ is odd, then

$$
\begin{aligned}
& \sigma\left(\Gamma_{2 p+1}\right) \\
& =\left\{\begin{array}{c}
1+\sum_{k=1}^{(p-1) / 3}\left(C_{p-k}^{2 k-1}+C_{p-k}^{2 k}\right) \\
+\sum_{k=1}^{(p+2) / 3}\left(C_{p-k}^{2 k-2}+C_{p-k}^{2 k-1}\right) \quad \text { if } n \equiv 0 \bmod 3 ; \\
1+\sum_{k=1}^{p / 3}\left(C_{p-k}^{2 k-1}+C_{p-k}^{2 k}\right) \\
+\sum_{k=1}^{p / 3}\left(C_{p-k}^{2 k-2}+C_{p-k}^{2 k-1}\right) \\
1+\sum_{k=1}^{(p+1) / 3}\left(C_{p-k}^{2 k-1}+C_{p-k}^{2 k}\right) \\
+\sum_{k=1}^{(p+1) / 3}\left(C_{p-k}^{2 k-2}+C_{p-k}^{2 k-1}\right)
\end{array} \quad \text { if } n \equiv 2 \bmod 3 .\right.
\end{aligned}
$$

(ii) If $n(n=2 p)$ is even, then

$$
\begin{aligned}
& \sigma\left(\Gamma_{2 p}\right) \\
& =\left\{\begin{array}{c}
1+\sum_{k=1}^{p / 3}\left(C_{p-k}^{2 k-2}+C_{p-k}^{2 k-1}\right) \\
+\sum_{k=2}^{(p+3) / 3}\left(C_{p-k}^{2 k-3}+C_{p-k}^{2 k-2}\right) \quad \text { if } n \equiv 0 \bmod 3 ; \\
1+\sum_{k=1}^{(p+1) / 3}\left(C_{p-k}^{2 k-2}+C_{p-k}^{2 k-1}\right) \\
+\sum_{k=2}^{(p+1) / 3}\left(C_{p-k}^{2 k-3}+C_{p-k}^{2 k-2}\right) \\
1+\sum_{k=1}^{(p+2) / 3}\left(C_{p-k}^{2 k-2}+C_{p-k}^{2 k-1}\right) \\
+\sum_{k=2}^{(p+2) / 3}\left(C_{p-k}^{2 k-3}+C_{p-k}^{2 k-2}\right)
\end{array} \text { if } n \equiv 2 \bmod 3 ;\right.
\end{aligned}
$$

Proof. We prove only that the theorem is correct if $n$ ( $n=$ $2 p+1)$ is odd and $n \equiv 0 \bmod 3$. And the proofs of the others cases are similar. End-vertices of $\Gamma_{n}$ can be divided into two cases as follows.

Case 1. The end-vertices set is composed of end-vertices with strings form $1 b_{2} b_{3} \cdots b_{n}$.

Case 2. The end-vertices set is composed of end-vertices with strings form $0 b_{2} b_{3} \cdots b_{n}$.

In Case 1 , since $n=2 p+1$ and $n \equiv 0 \bmod 3$, then endvertices $\left\{u_{n, k, z}\right\}$ are divided into $((4 p-1) / 3)+1$ cases with $z=$ $0,1,2, \ldots,(4 p-1) / 3$. Therefore the number of end-vertices with strings form $1 b_{2} b_{3} \cdots b_{n}$ is

$$
1+\sum_{k=1}^{(p-1) / 3}\left(C_{p-k}^{2 k-1}+C_{p-k}^{2 k}\right)
$$

In Case 2, as similar as Case 1, end-vertices $\left\{u_{n, k, z}\right\}$ are divided into $((4 p+2) / 3)+1$ cases with $z=0,1,2, \ldots,(4 p+$ $2) / 3$. Then the number of end-vertices with strings form $0 b_{2} b_{3} \cdots b_{n}$ is

$$
\sum_{k=1}^{(p+2) / 3}\left(C_{p-k}^{2 k-2}+C_{p-k}^{2 k-1}\right)
$$

Therefore

$$
\begin{aligned}
\sigma\left(\Gamma_{2 p+1}\right)= & +\sum_{k=1}^{(p-1) / 3}\left(C_{p-k}^{2 k-1}+C_{p-k}^{2 k}\right) \\
& +\sum_{k=1}^{(p+2) / 3}\left(C_{p-k}^{2 k-2}+C_{p-k}^{2 k-1}\right) n \equiv 0 \bmod 3 .
\end{aligned}
$$

This completes our proof.

Now we give the larger bound on the domination number of Fibonacci cubes as follows.

Theorem 5. Let $n>4$. Then for the Fibonacci cube $\Gamma_{n}$ the followings hold.

(i) If $n(n=2 p+1)$ is odd, then

$$
\begin{aligned}
& {\left[\frac{F_{n}-3}{n-2}\right]} \\
& \leq \gamma\left(\Gamma_{n}\right) \\
& \leq\left\{\begin{array}{c}
2^{p}+2+\sum_{k=1}^{(p-1) / 3}\left(C_{p-k}^{2 k-1}+C_{p-k}^{2 k}\right)\left(2^{p-k}-1\right) \\
+\sum_{k=1}^{(p+2) / 3}\left(C_{p-k}^{2 k-2}+C_{p-k}^{2 k-1}\right)\left(2^{p-k}-1\right) \\
+\sum_{k=1}^{p / 3}\left(C_{p-k}^{2 k-2}+C_{p-k}^{2 k-1}\right)\left(2^{p-k}-1\right) \\
\text { if } n \equiv 0 \bmod 3 ; \\
2^{p}+2+\sum_{k=1}^{p / 3}\left(C_{p-k}^{2 k-1}+C_{p-k}^{2 k}\right)\left(2^{p-k}-1\right) \\
+\sum_{k=1}^{(p+1) / 3}\left(C_{p-k}^{2 k-2}+C_{p-k}^{2 k-1}\right)\left(2^{p-k}-1\right) \\
\text { if } n \equiv 2 \bmod 3 .
\end{array}\right.
\end{aligned}
$$


(ii) If $n(n=2 p)$ is even, then

$$
\begin{aligned}
& {\left[\frac{F_{n}-3}{n-2}\right]} \\
& \leq \gamma\left(\Gamma_{n}\right) \\
& \leq\left\{\begin{array}{c}
2^{p-1}+2+\sum_{k=1}^{p / 3}\left(C_{p-k}^{2 k-2}+C_{p-k}^{2 k-1}\right)\left(2^{p-k}-1\right) \\
+\sum_{k=2}^{(p+3) / 3}\left(C_{p-k}^{2 k-3}+C_{p-k}^{2 k-2}\right)\left(2^{p-k}-1\right) \\
2^{p-1}+2+\sum_{k=1}^{(p+1) / 3}\left(C_{p-k}^{2 k-2}+C_{p-k}^{2 k-1}\right)\left(2^{p-k}-1\right) \\
+\sum_{k=2}^{(p+1) / 3}\left(C_{p-k}^{2 k-3}+C_{p-k}^{2 k-2}\right)\left(2^{p-k}-1\right) \\
2^{p-1}+2+\sum_{k=1}^{(p+2) / 3}\left(C_{p-k}^{2 k-2}+C_{p-k}^{2 k-1}\right)\left(2^{p-k}-1\right) \\
+\sum_{k=2}^{(p+2) / 3}\left(C_{p-k}^{2 k-3}+C_{p-k}^{2 k-2}\right)\left(2^{p-k}-1\right) \\
\text { if } n \equiv 2 \bmod 3 .
\end{array}\right.
\end{aligned}
$$

Proof. According to the process of proof of Lemma 3, there must exist a dominating set of $\Gamma\left[u_{n, k, z}\right]$ with the string form $1 b_{2} \cdots b_{n}$ (or $0 b_{2} \cdots b_{n}$ ) such that it contains the vertex with the string form $10^{n-1}$ (or $010^{n-2}$ ). So all dominating sets of $\Gamma\left[u_{n, k, z}\right]$ of $\Gamma_{n}$ with strings form $1 b_{2} \cdots b_{n}$ (or $0 b_{2} \cdots b_{n}$ ) have at least two common vertices with strings $\left\{10^{n-1}, 0^{n}\right\}$ (or $\left\{010^{n-2}, 0^{n}\right\}$ ). Then the theorem follows directly from Lemmas 2 and 3 and Theorem 4.

\section{The Larger Bound on the Domination Number of Lucas Cubes}

In this section, we will determine the larger bound on the domination number of Lucas cubes as follows.

Lemma 6 (see [3]). For any $n \geq 7, \gamma\left(\Lambda_{n}\right) \geq\left\lceil\left(L_{n}-2 n\right) /(n-3)\right\rceil$.

Theorem 7. Let $\sigma\left(\Lambda_{n}\right)$ be the number of end-vertices in $\Lambda_{n}$. Then the followings hold.

(i) If $n(n=2 p+1)$ is odd, then

$$
\begin{aligned}
& \sigma\left(\Lambda_{2 p+1}\right) \\
& = \begin{cases}\sum_{k=1}^{(p-1) / 3} C_{p-k}^{2 k-1}+\sum_{k=1}^{p / 3}\left(C_{p-k}^{2 k-2}+C_{p-k}^{2 k-1}\right) & \text { if } n \equiv 0 \bmod 3 ; \\
\sum_{k=1}^{p / 3} C_{p-k}^{2 k-1}+\sum_{k=1}^{p / 3}\left(C_{p-k}^{2 k-2}+C_{p-k}^{2 k-1}\right) & \text { if } n \equiv 1 \bmod 3 ; \\
\sum_{k=1}^{(p+1) / 3} C_{p-k}^{2 k-1}+\sum_{k=1}^{(p+1) / 3}\left(C_{p-k}^{2 k-2}+C_{p-k}^{2 k-1}\right) & \text { if } n \equiv 2 \bmod 3 .\end{cases}
\end{aligned}
$$

(ii) If $n(n=2 p)$ is even, then

$$
\begin{aligned}
& \sigma\left(\Lambda_{2 p}\right)
\end{aligned}
$$

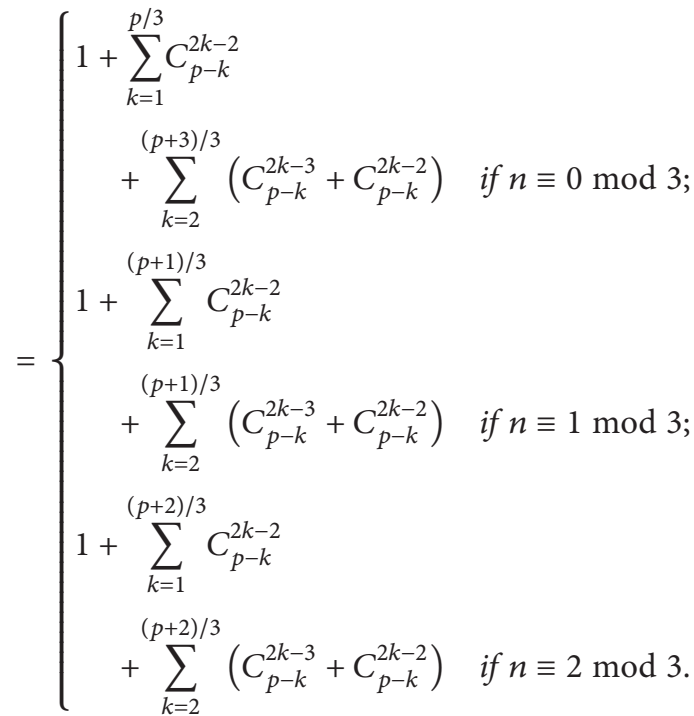

Proof. The proof is similar to Theorem 4.

Theorem 8. Let $n>$ 7. Then for the Lucas cube $\Lambda_{n}$ the followings hold.

(i) If $n(n=2 p+1)$ is odd, then

$\left\lceil\frac{L_{n}-2 n}{n-3}\right\rceil$

$\leq \gamma\left(\Lambda_{n}\right)$

$$
\leq\left\{\begin{array}{l}
2+\sum_{k=1}^{(p-1) / 3} C_{p-k}^{2 k-1}\left(2^{p-k}-1\right) \\
+\sum_{k=1}^{(p+2) / 3}\left(C_{p-k}^{2 k-2}+C_{p-k}^{2 k-1}\right)\left(2^{p-k}-1\right) \quad \text { if } n \equiv 0 \bmod 3 \\
2+\sum_{k=1}^{p / 3} C_{p-k}^{2 k-1}\left(2^{p-k}-1\right) \\
+\sum_{k=1}^{p / 3}\left(C_{p-k}^{2 k-2}+C_{p-k}^{2 k-1}\right)\left(2^{p-k}-1\right) \\
+\sum_{k=1}^{(p+1) / 3} C_{p-k}^{2 k-1}\left(2^{p-k}-1\right) \\
+\sum_{k=1}^{(p+1) / 3}\left(C_{p-k}^{2 k-2}+C_{p-k}^{2 k-1}\right)\left(2^{p-k}-1\right) \quad \text { if } n \equiv 1 \bmod 3
\end{array}\right.
$$


(ii) If $n(n=2 p)$ is even, then

$$
\begin{aligned}
& {\left[\frac{L_{n}-2 n}{n-3}\right\rceil} \\
& \leq \gamma\left(\Lambda_{n}\right) \\
& \leq\left\{\begin{array}{c}
2^{p-1}+2+\sum_{k=1}^{p / 3} C_{p-k}^{2 k-2}\left(2^{p-k}-1\right) \\
+\sum_{k=2}^{(p+3) / 3}\left(C_{p-k}^{2 k-3}+C_{p-k}^{2 k-2}\right)\left(2^{p-k}-1\right) \quad \text { if } n \equiv 0 \bmod 3 ; \\
2^{p-1}+2+\sum_{k=1}^{(p+1) / 3} C_{p-k}^{2 k-2}\left(2^{p-k}-1\right) \\
+\sum_{k=2}^{(p+1) / 3}\left(C_{p-k}^{2 k-3}+C_{p-k}^{2 k-2}\right)\left(2^{p-k}-1\right) \quad \text { ifn } \equiv 1 \bmod 3 ; \\
2^{p-1}+2+\sum_{k=1}^{(p+2) / 3} C_{p-k}^{2 k-2}\left(2^{p-k}-1\right) \\
\quad+\sum_{k=2}^{(p+2) / 3}\left(C_{p-k}^{2 k-3}+C_{p-k}^{2 k-2}\right)\left(2^{p-k}-1\right) \quad \text { if } n \equiv 2 \bmod 3 .
\end{array}\right.
\end{aligned}
$$

Proof. That follows directly from Lemmas 6 and Theorem 7.

\section{Conflict of Interests}

The author declares that there is no conflict of interests regarding the publication of this paper.

\section{Acknowledgments}

The author is very grateful to Professor Wenpeng Zhang for useful suggestions and the two referees for valuable comments. This work is supported by TSNC of China (TSA1013).

\section{References}

[1] W.-J. Hsu, "Fibonacci cubes. A new interconnection topology," IEEE Transactions on Parallel and Distributed Systems, vol. 4, no. 1, pp. 3-12, 1993.

[2] E. Dedó, D. Torri, and N. Z. Salvi, "The observability of the Fibonacci and the Lucas cubes," Discrete Mathematics, vol. 255, no. 1-3, pp. 55-63, 2002.

[3] A. Castro, S. Klavžar, M. Mollard, and Y. Rho, "On the domination number and the 2-packing number of Fibonacci cubes and Lucas cubes," Computers \& Mathematics with Applications, vol. 61, no. 9, pp. 2655-2660, 2011.

[4] A. Castro and M. Mollard, "The eccentricity sequences of Fibonacci and Lucas cubes," Discrete Mathematics, vol. 312, no. 5, pp. 1025-1037, 2012.

[5] E. Munarini and N. Z. Salvi, "Structural and enumerative properties of the Fibonacci cubes," Discrete Mathematics, vol. 255, no. 1-3, pp. 317-324, 2002.

[6] D. A. Pike and Y. Zou, "The domination number of fibonacci cubes," Journal of Combinatorial Mathematics and Combinatorial Computing, vol. 80, pp. 433-444, 2012.
[7] C. Whitehead and N. Z. Salvi, "Observability of the extended Fibonacci cubes," Discrete Mathematics, vol. 266, no. 1-3, pp. 431-440, 2003.

[8] J. A. Bondy and U. S. R. Murty, Graph Theory with Applications, Macmillan, London, UK; Elsevier, New York, NY, USA, 1976.

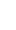




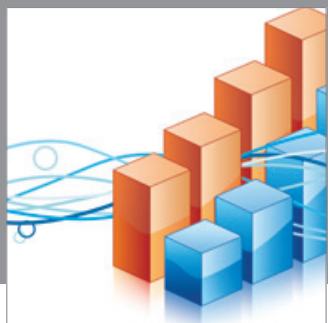

Advances in

Operations Research

mansans

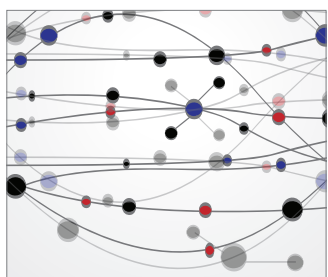

The Scientific World Journal
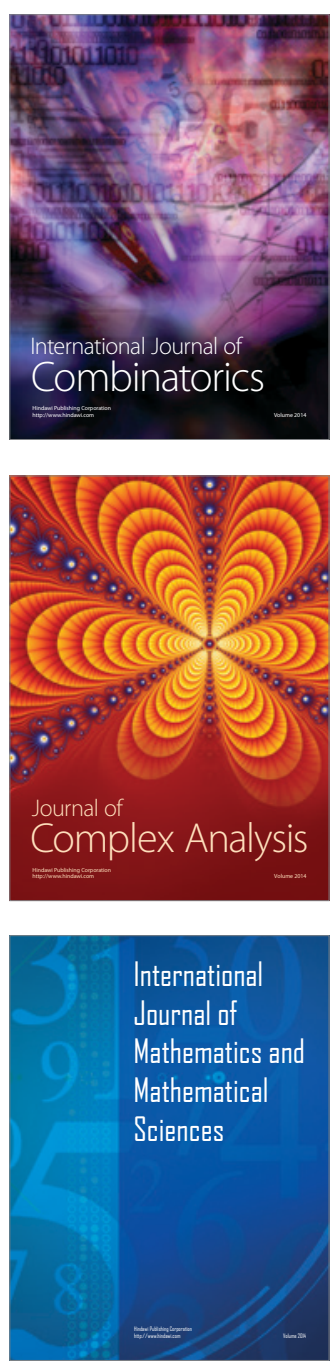
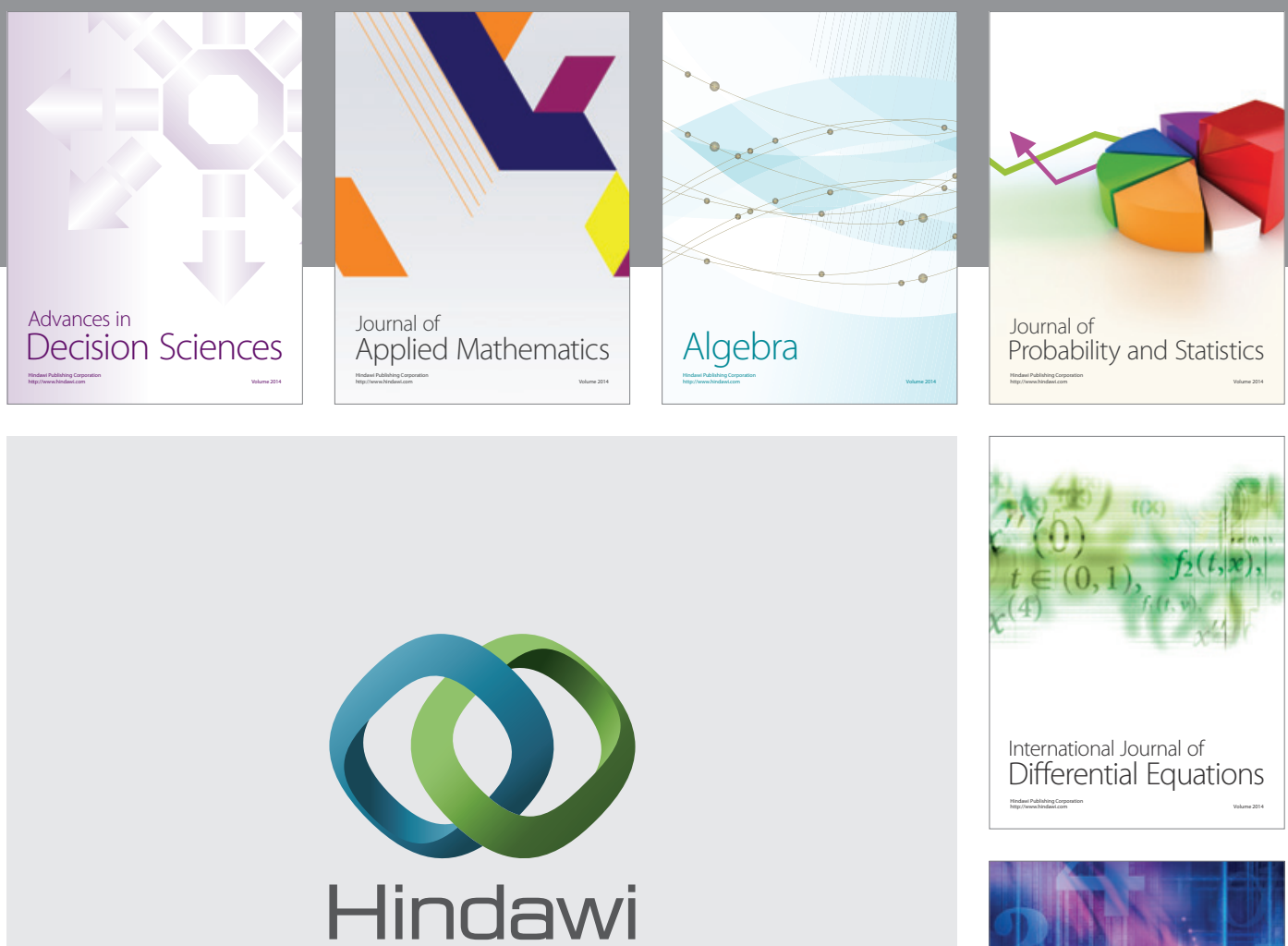

Submit your manuscripts at http://www.hindawi.com
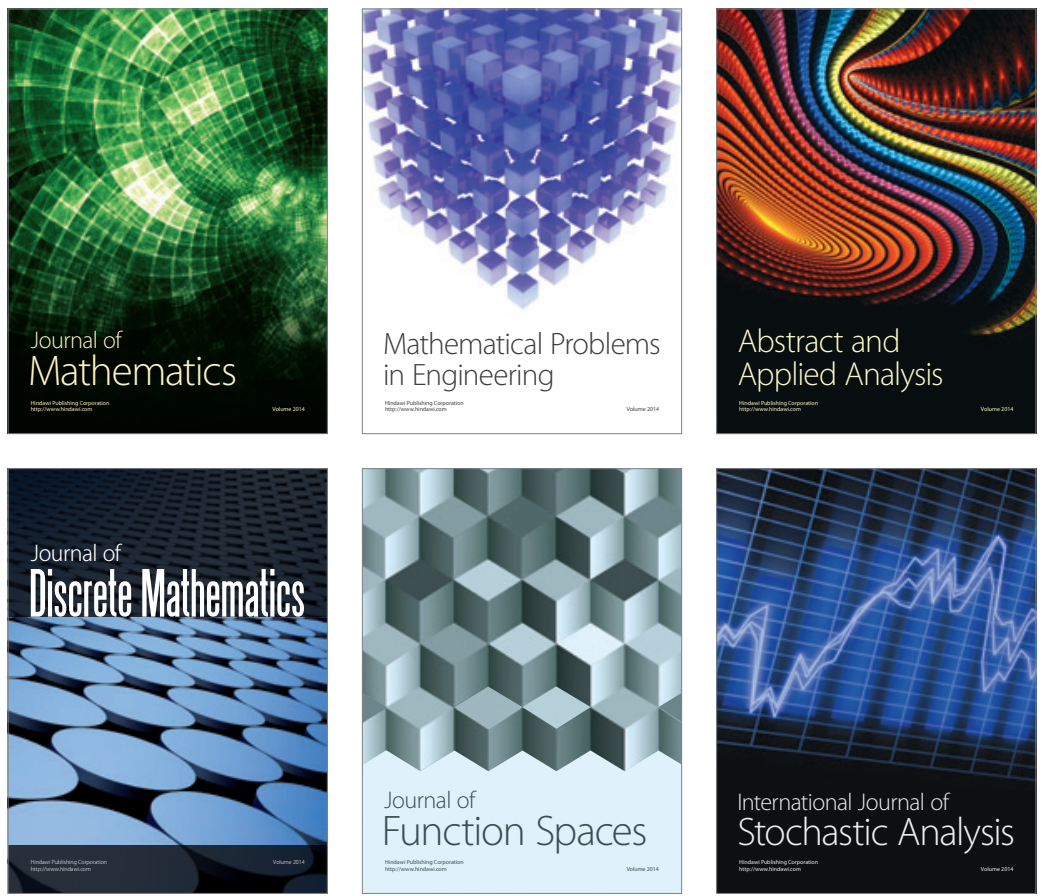

Journal of

Function Spaces

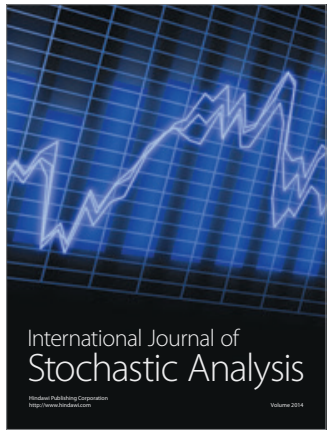

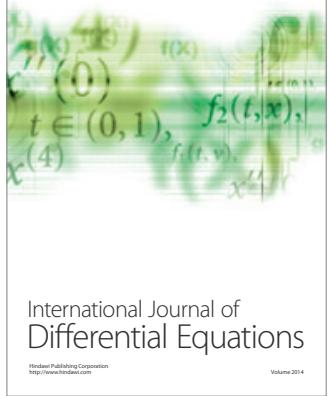
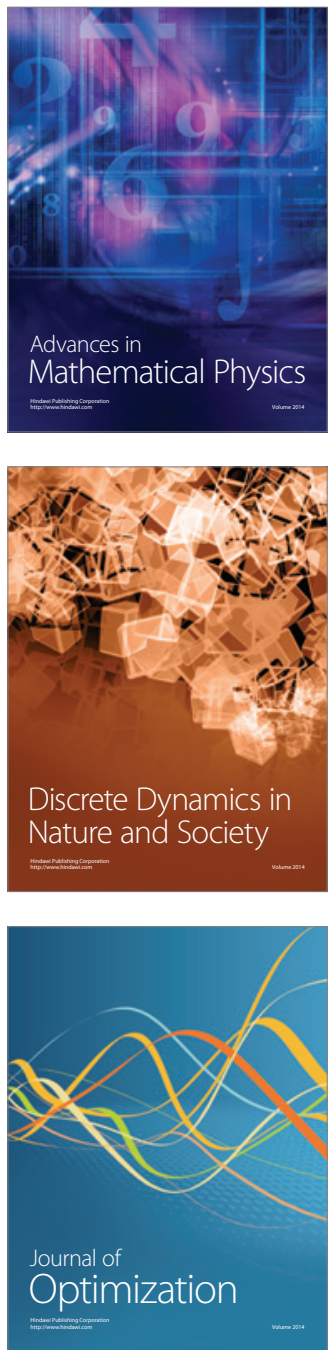